SHORT REPORT

\title{
A lteration of the cortical motor map in a patient with intractable focal seizures
}

\author{
F A Lado, A D Legatt, P A LaSala, S Shinnar
}

J N eurol N eurosurg Psychiatry 2002;72:812-815

Patients with epilepsia partialis continua may develop progressive neurological deficits of unclear origin. It is possible that repetitive epileptic spikes induce plastic changes in the cortex analogous to the changes observed following direct microstimulation. A child is reported with focal cortical dysplasia, intractable focal seizures, worsening hemiparesis, and alteration of the cortical motor map over time. At age 7 , he underwent cortical motor mapping before partial resection of a seizure focus within the right postcentral gyrus. No deficits were present after surgery, and seizure frequency declined by more than $90 \%$. Seizures subsequently worsened and a progressive left hemiparesis developed. Cortical remapping at age 12 showed motor centres for left arm, face, and eye movements in an unusual configuration. The location of the motor representation of the face differed from the location obtained at age 7 . This case provides direct electrophysiological evidence of reorganisation of the cortical motor map in the human brain.

$\mathrm{T}$ he plasticity of topographic representations of sensory and motor functions is a fundamental characteristic of the cortex that may be altered by changes in peripheral or central activation. Peripheral denervation can result in "unmasking" of adjacent motor and sensory somatotopic representations. ${ }^{1-3}$ In the human cortex, somatotopic remapping in the motor and somatosensory cortex follows changes in peripheral innervation. ${ }^{4-7}$ Increased peripheral input or even repetitive microstimulation applied directly to the cortex expands the cortical area representing a particular motor or sensory function..$^{8-10}$

In animals and humans, recurrent or prolonged seizures are associated with hippocampal mossy fibre sprouting. ${ }^{11}{ }^{12}$ Comparatively little is known of the functional consequences of extratemporal epileptiform activity. In particular, the effects of repetitive epileptic spikes on neocortical topographical representations are unknown. Earlier case reports have linked focal cortical dysplasia and epilepsia partialis continua with progressive hemiparesis. ${ }^{13-16}$ Neurosurgeons are familiar with alterations of the cortical map resulting from proliferative or necrotising lesions. We report a patient with focal cortical dysplasia and epilepsia partialis continua originating in the sensorimotor cortex, in whom the topographic organisation of the motor cortex changed between two cortical mapping studies performed five years apart. Our case is unique in that the change in the cortical map correlated with the emergence of progressive neurological deficits despite the absence of a progressive structural lesion.

\section{CASE REPORT}

The patient is a 12 year old boy with intractable partial seizures. Birth and development were normal. At age seven, simple focal seizures developed, affecting the left side of the face. Magnetic resonance imaging (MRI) identified an abnormal signal in the posterior right frontal cortex (fig 1). Focal seizures worsened and progressed to epilepsia partialis continua despite treatment. The patient was admitted for resection of epileptogenic cortex. There was no facial weakness, hemiparesis, or somatosensory impairment at this time. Intraoperative electrocorticography identified a postcentral seizure focus, and a small focal resection limited to the postcentral gyrus was performed. Postoperatively, there was a greater than $90 \%$ reduction in seizure frequency, without neurological deficit. Pathological examination revealed focal cortical dysplasia.

Over five years, seizure frequency increased again to epilepsia partialis continua. Even more ominously, a progressive subtle left hemiparesis and cognitive impairment developed. At age 12, the patient was admitted to hospital for further resection after localisation of seizure onset and motor mapping. The patient's seizures were sufficiently disabling to justify the risk of producing a neurological deficit. A more extensive resection, including the pre- and postcentral epileptogenic cortex, was performed. The pathology was diffuse cortical dysplasia (fig 2). Postoperative MRI confirmed the extent of the resection. A dense hemiparesis present immediately after surgery has progressively improved. At his last follow up visit, one year post-surgery, the patient walks freely with a brace, has good use of the left hand, and minimal facial weakness. Cognitive function has markedly improved and seizures are simple partial, brief, and occur less than once a week.

\section{Electrocorticography and sensory/motor mapping}

Electroclinical seizures originated at multiple subdural electrodes. During the first surgery at age seven, a subdural grid was positioned intraoperatively over the radiographic abnormality. Frequent electroclinical seizures arose at electrode A13, even under general anaesthesia (fig 3B). The Rolandic sulcus was identified by the polarity reversal of the SSEP (fig 3A) and the effect of electrical cortical stimulation (fig 3C). The full extent of the SSEP was not completely represented in the area covered by the recording grid or within the craniotomy. As the SSEP was necessary only to determine the anteroposterior location of the central sulcus, a larger craniotomy was not indicated. The most active seizure focus was postcentral (fig 3B). ${ }^{18}$ Stimulation of the precentral gyrus $1 \mathrm{~cm}$ anterior to the seizure focus produced left facial movements (fig 3C). An area of cortex of around $1.5 \mathrm{~cm}$ diameter posterior to the Rolandic sulcus was resected (dotted circle in fig 3B).

At age 12, the patient received an implanted subdural electrode array exceeding the cortical area covered in the first surgery (fig $3 \mathrm{D}, \mathrm{E}$ ). The locations of the cortical scar from the first surgery, the Sylvian fissure, and the Rolandic sulcus guided grid placement in the second surgery. The same surgeon and neurophysiologist participated in both operations. Electrocorticography and motor mapping were performed extraoperatively for several days without anaesthesia effects. Frequent spikes and seizures originated at multiple electrodes (fig 3D); 

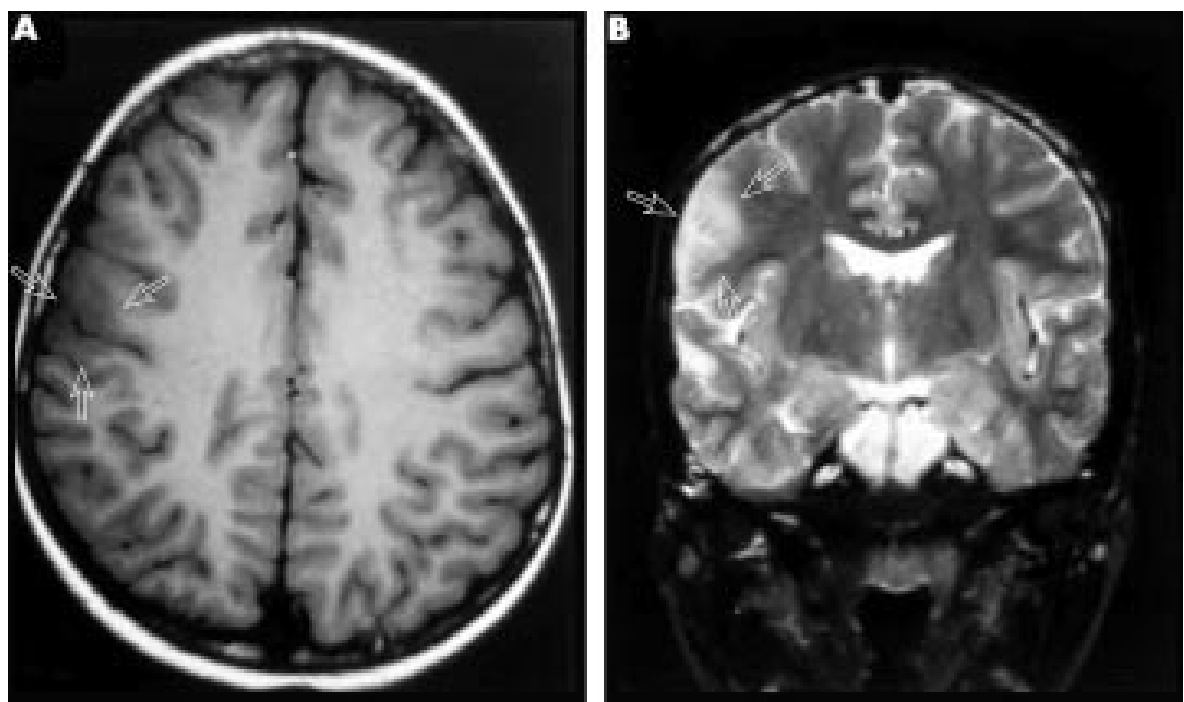

Figure 1 Magnetic resonance images created with a 1.5 T magnet scanner before the patient's first surgery. (A) T-1 weighted axial image showing a subtle signal abnormality in the right posterior frontal-central region (arrowed). (B) T-2 weighted frontal image showing abnormal signal intensity in the cortex superior to the Sylvian fissure (arrowed).

the most active seizure foci were precentral. Electrical cortical stimulation produced eye, face, or arm movements at multiple electrodes in the superior portion of the implant (fig 3E). The boundaries of the second cortical resection were based on the epileptogenic and functional characteristics of the underlying cortex. Comparison of electrocorticography and stimulation results obtained in the first and second mappings showed the following:

- Stimulation of the cortical region that produced facial twitching in the presence of general anaesthesia in the first surgery no longer produced any movement at the time of the second surgery (fig 3C, E).

- At the time of the second surgery, the somatotopic motor representation of the face was at an unusual location, displaced towards the vertex (fig $3 \mathrm{E}$ ). The facial motor representation typically lies closer to the Sylvian fissure, inferior to the arm representation. ${ }^{19}$
- There is an interval change in the location of the epileptogenic zone. The main seizure focus shifted anteriorly across the Rolandic sulcus (fig 3B, D). The extent of epileptogenic zone cannot be compared as the first electrocorticography was performed under general anaesthesia.

\section{DISCUSSION}

This patient developed progressive neurological and cognitive deficits associated with an interval change in the cortical motor representation, abnormal motor representation of the face, and a shift in the main seizure focus across the Rolandic sulcus in the five year interval between operations. The change in the cortical motor representation may represent a relative rather than total loss of function in a previously functional cortex. However, this change cannot be attributed to an ongoing progressive or proliferative structural lesion, because focal cortical dysplasia is a static congenital lesion. The presence of
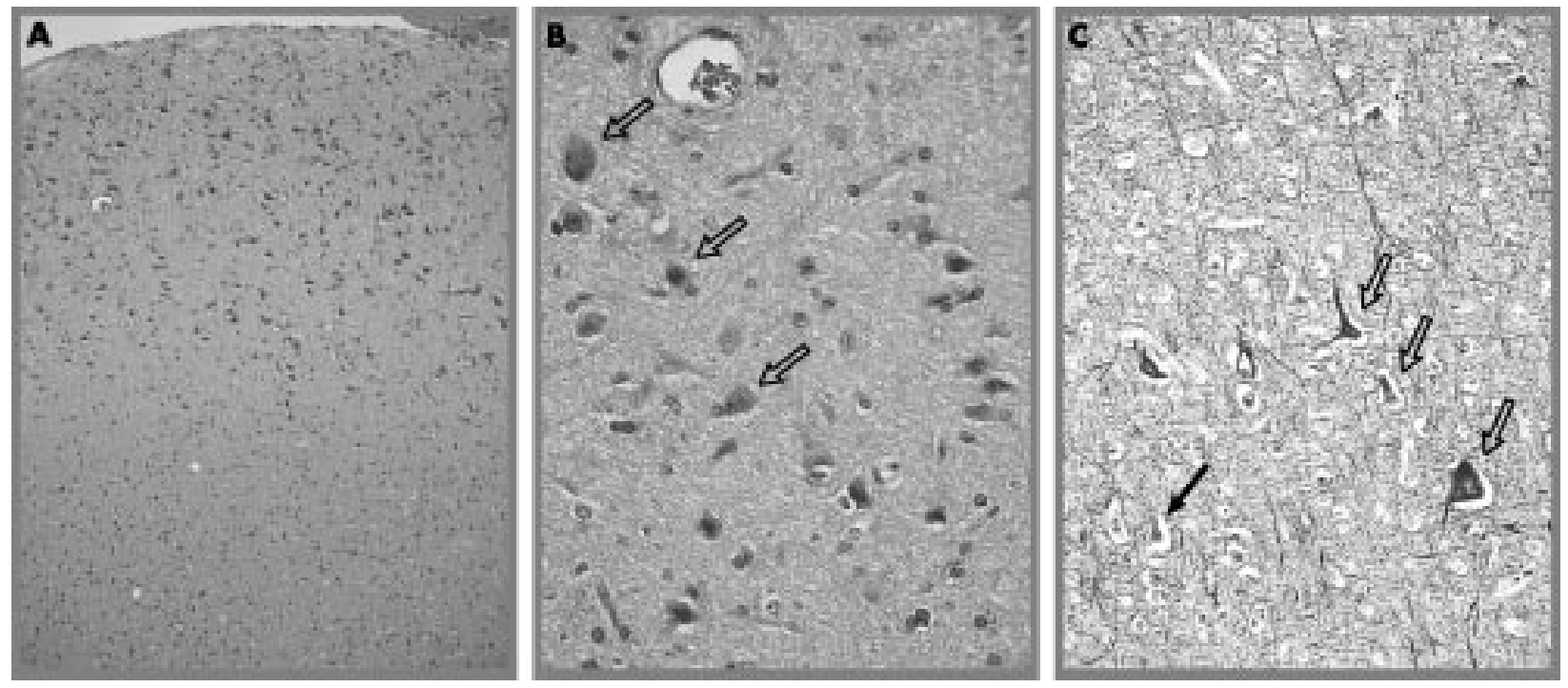

Figure 2 Photomicrographs taken following second surgery showing focal aggregates of large ganglion cells without the usual cortical lamination. (A) At 10x magnification there is a loss of typical laminar organisation of cortex; large "balloon" ganglion cells are oriented randomly, an appearance characteristic of focal cortical dysplasia; (B) $40 \times$ magnification view showing focal aggregates of "balloon" ganglion cells; (C) $20 \times$ magnification silver stain of a large ganglion cell showing an abnormally large dendrite. 
A

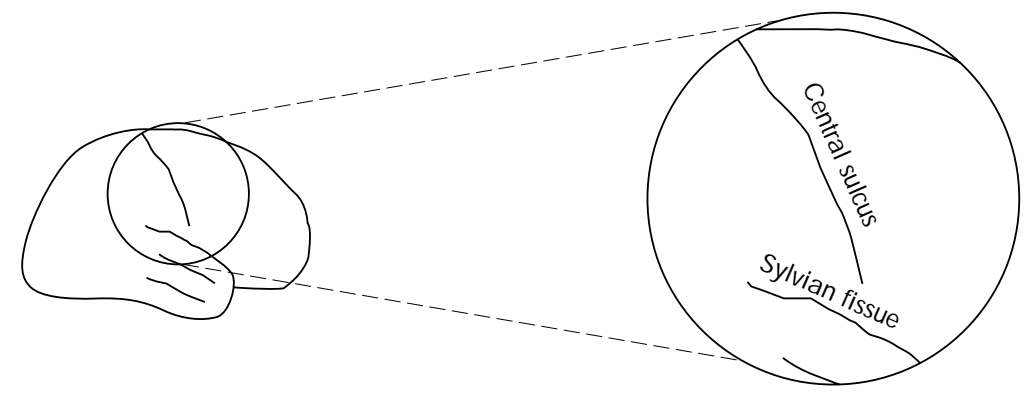

Electrocorticography results

B

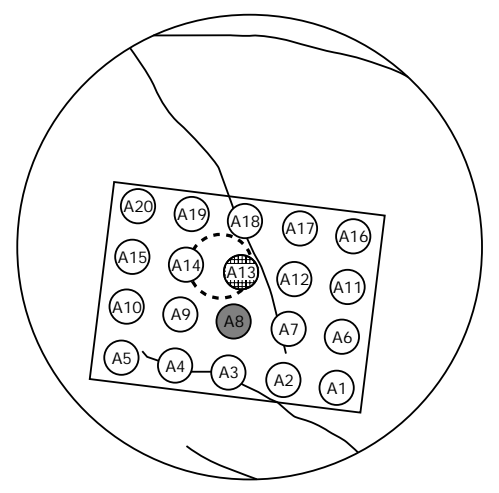

D

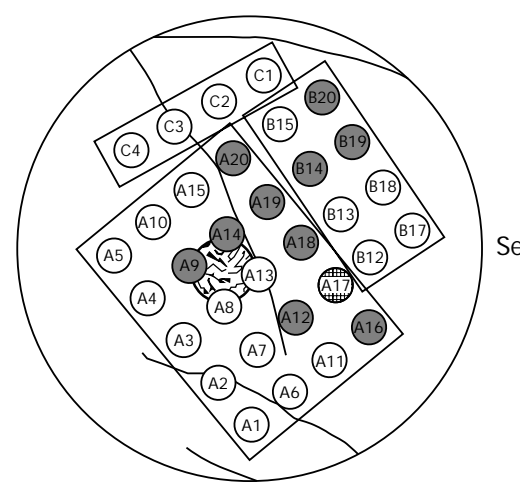

Cortical stimulation results

C

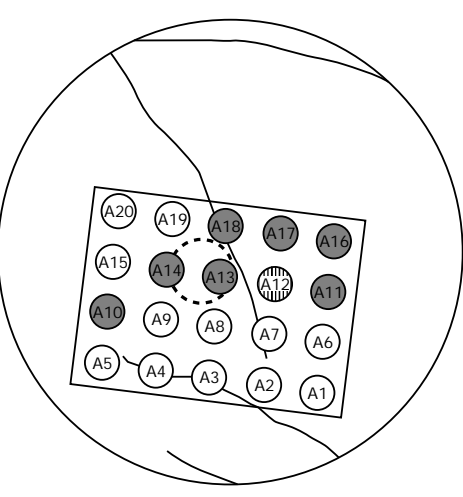

E

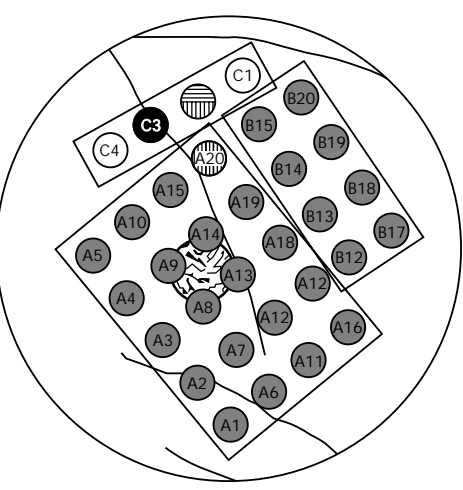

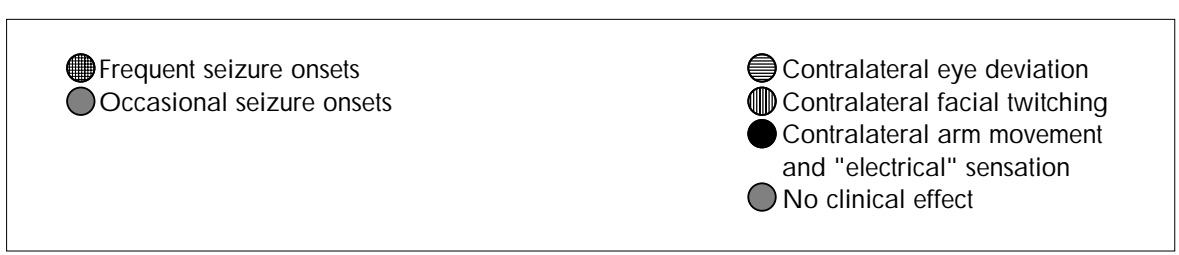

Figure 3 (A) Schematic diagram indicating the location of anatomical landmarks shown in subsequent figures. Identification of the Rolandic sulcus was based on the location of the polarity reversal of the median nerve somatosensory evoked potential. (B) Seizure onset areas identified under general anaesthesia during the first surgery are indicated (left). A dashed circle marks the area that was subsequently resected. Before the second surgery, seizure onset areas were identified during extraoperative recordings from implanted electrodes in the unanaesthetised state (right). (C) Motor mapping results produced by electrical stimulation under general anaesthesia during the first surgery are shown (left). Results of extraoperative electrical stimulation of cortex show a shift in the motor map compared to the first surgery (right). In (B) and (C), a stippled circle indicates the area of the earlier resection.

focal cortical dysplasia in the region of altered motor representation, however, raises the possibility that the motor map was abnormal before the first surgery. While this possibility cannot be completely addressed with the available data, we note that the motor mapping from the first surgery established that the motor representation of the face was at an expected location.
We believe that the change in motor representation and the development of hemiparesis over five years most probably resulted either from the effects of the postcentral lesion on the precentral motor map or from alteration of precentral synapses by continuous focal spiking in motor cortex. The change in motor representation might also have arisen from progressive gradual neuronal loss in the motor cortex. We 
discount this possibility because progressive neuronal loss is not typical of focal cortical dysplasia and was not apparent on pathological examination at the second operation.

The motor cortex has reciprocal connections with the postcentral somatosensory cortex. ${ }^{2021}$ A lesion in the postcentral gyrus could result in a relative deafferentation of motor cortex. In animals, a somatosensory lesion alters and disorganises adjacent somatosensory receptive fields.[22] To our knowledge the effects of somatosensory lesions on the motor cortex have not been extensively studied. The change in the motor map, however, was not the result of precentral damage. In the first operation, the precentral gyrus was localised and preserved intraoperatively and motor deficits were absent postoperatively.

We believe it is likely that unremitting focal epileptic discharges induced plastic synaptic changes in nearby cortex. Use dependent changes in synaptic efficiency-that is, Hebbian learning-in the neocortex and hippocampus are important in learning and memory. ${ }^{23}$ The face area of this patient's right sensorimotor region produced almost continuous focal epileptic activity for long periods of time. We speculate that continuous focal paroxysmal activity altered neighbouring synapses in a Hebbian manner and disrupted physiological synaptic connectivity. Transcranial magnetic stimulation in patients with focal epilepsy has shown abnormal inhibition in the epileptic hemisphere, suggesting altered synaptic function. ${ }^{24}$ Physiological stimulation of the somatosensory cortex through peripheral receptors alters the sensory map by Hebbian synaptic mechanisms. ${ }^{25}$ Repetitive focal electrical stimulation of motor cortex-analogous to continuous epileptic spiking-produces expansion of the motor representation of the stimulated cortex. ${ }^{9} 10$

In our patient, Hebbian recruitment of the neighbouring cortex by an epileptic focus could expand the area of dysfunctional epileptogenic cortex, alter the motor map, and produce a gradual and progressive hemiparesis. In other words, the cortical "representation" of the seizure focus expanded over time, crowding and distorting the motor map. Resection of dysfunctional cortex at the second surgery abolished any residual normal cortical function in this region and worsened the postoperative hemiparesis. Resolution of postoperative brain oedema and inflammation produced some improvement, but the deficit remained worse postoperatively than preoperatively. While conclusions based on a single example are necessarily speculative, this case offers electrophysiological evidence of reorganisation in human motor cortex attributable either to ongoing focal epileptic activity or to focal lesioning of the postcentral gyrus.

\section{ACKNOWLEDGEMENTS}

We thank Dr Solomon L Moshé for helpful discussions and comments on the manuscript. Supported in part by NIH grant 1K08-NS41340 to FAL.

\section{Authors' affiliations}

F A Lado, A D Legatt, P A LaSala, S Shinnar, Comprehensive Epilepsy $M$ anagement Center, M ontefiore M edical Center, Bronx, N ew York, USA

Correspondence to: Dr F A Lado, Department of N eurology, K313,
Albert Einstein College of M edicine, 1410 Pelham Parkway South, Bronx NY 10461, USA; ladof@ix.netcom.com

Received 210 ctober 2000

In revised form 27 December 2001

Accepted 15 January 2002

\section{REFERENCES}

1 Donoghue JP. Plasticity of adult sensorimotor representations. Curr 0 pin N eurobiol 1995;5:749-54.

2 Merzenich MM, N elson RJ, Stryker M P, et al. Somatosensory cortical map changes following digit a mputation in adult monkeys. I Comp $N$ eurol 1984;224:591-605.

3 Sanes JN, Suner S, Lando JF, et al. Rapid reorganization of adult rat motor cortex somatic representation patterns after motor nerve injury. Proc $N$ atl Acad Sci USA 1988;85:2003-7.

4 Ziemann U, Hallett M, Cohen LG. M echanisms of deafferentation-induced plasticity in human motor cortex. I N eurosci $1998 ; 18: 7000-7$

5 Druschky K, Kaltenhauser M, Hummel C, et al. Alteration of the somatosensory cortical map in peripheral mononeuropathy due to carpal tunnel syndrome. N euroreport 2000;11:3925-30.

6 Ribary U, Cappell J, Mogilner A, et al. Functional imaging of plastic changes in the human brain. Adv N eurol 1999;81:49-56.

7 Ojemann JG, Silbergeld DL. Cortical stimulation mapping of phantom limb rolandic cortex. Case report. J N eurosurg 1995;82:641-4.

8 Jenkins WM, M erzenich M M, 0 chs MT, et al. Functional reorganization of primary somatosensory cortex in adult ow I monkeys after behaviorally controlled tactile stimulation. J N europhysiol 1990;63:82-104.

9 Recanzone GH, M erzenich M M. Dinse HR. Expansion of the cortical representation of a specific skin field in primary somatosensory cortex by intracortical microstimulation. Cereb Cortex 1992;2:181-96

10 Nudo RJ, Jenkins W M, M erzenich M M. Repetitive microstimulation alters the cortical representation of movements in adult rats. Somatosens $M$ ot Res 1990;7:463-83.

11 Cavazos JE, Golarai G, Sutula TP. Mossy fiber synaptic reorganization induced by kindling: time course of development, progression, and permanence. I Neurosci 1991;11:2795-803.

12 Sutula T, Cascino G, Cavazos J, et al. Mossy fiber synaptic reorganization in the epileptic human temporal lobe. Ann $\mathrm{N}$ eurol $1989 ; 26: 321-30$.

13 Fusco L, Bertini E, Vigevano F. Epilepsia partialis continua and neuronal migration anomalies. Brain Dev 1992;14:323-8.

14 Kuzniecky R, Powers R. Epilepsia partialis continua due to cortical dysplasia. J Child N eurol 1993;8:386-8.

15 Andermann F. Epilepsia partialis continua and other seizures arising from the precentral gyrus: high incidence in patients with Rasmussen syndrome and neuronal migration disorders. Brain Dev 1992;14:338-9.

16 Palmini A, Andermann F, O livier A, et al. Focal neuronal migration disorders and intractable partial epilepsy: a study of 30 patients. Ann $N$ eurol 1991;30:741-9.

17 Legatt A. Intraoperative neurophysiologic monitoring. In: Frost $E$, ed. Clinical anesthesia in neurosurgery, 2 nd ed. Stoneham, M assachusetts: Butterworths, 1991:63-127.

18 Legatt A, LaSala P, M itnick R, et al. Electrophysiologic studies and intraoperative localization in a child with epilepsia partialis continua. Epilepsy 1996;9:192-7.

19 Penfield W, Rasmussen T. The cerebral cortex of man. A clinical study of localization of function. $\mathrm{N}$ ew York: Macmillan, 1950.

20 Ghosh S, Brinkman C, Porter R. A quantitative study of the distribution of neurons projecting to the precentral motor cortex in the monkey ( $M$ fascicularis). J Comp N eurol 1987;259:424-44.

21 Leichnetz G. Afferent and efferent connections of the dorsolateral precentral gyrus (area 4, hand/ arm region) in the macaque monkey, with comparison to area 8. J Comp N eurol 1986;254:460-92

22 Doetsch GS, Johnston KW, Hannan CJ. Physiological changes in the somatosensory forepaw cerebral cortex of adult raccoons following lesions of a single cortical digit representation. Exp N eurol 1990:108:162-75.

23 Skrebitsky VG, Chepkova AN. Hebbian synapses in cortical and hippocampal pathways. Rev N eurosci 1998;9:243-64

24 Cicinelli P, M attia D, Spanedda F, et al. Transcranial magnetic stimulation reveals an interhemispheric a symmetry of cortical inhibition in focal epilepsy. N euroreport 2000;11:701-7.

25 Wang X, M erzenich M M, Sameshima K, et al. Remodelling of hand representation in adult cortex determined by timing of tactile stimulation. $N$ ature $1995 ; 378: 71-5$ 